Cinémas

Revue d'études cinématographiques

Journal of Film Studies

\title{
La femme et le type : le stéréotype comme vecteur narratif dans le cinéma des attractions
}

\section{Pierre Chemartin et Nicolas Dulac}

Volume 16, numéro 1, automne 2005

Femmes et cinéma muet : nouvelles problématiques, nouvelles méthodologies

URI : https://id.erudit.org/iderudit/013054ar

DOI : https://doi.org/10.7202/013054ar

Aller au sommaire du numéro

Éditeur(s)

Cinémas

ISSN

1181-6945 (imprimé)

1705-6500 (numérique)

Découvrir la revue

Citer cet article

Chemartin, P. \& Dulac, N. (2005). La femme et le type : le stéréotype comme vecteur narratif dans le cinéma des attractions. Cinémas, 16(1), 139-161. https://doi.org/10.7202/013054ar

\section{Résumé de l'article}

Le cinéma des premiers temps avait largement recours aux stéréotypes, comme en témoigne l'abondance, au sein des vues animées, de figures féminines figées et simplistes. Plutôt que de l'appréhender seulement comme reflet d'une subjectivité, ainsi que le propose l'approche féministe traditionnelle, le stéréotype mériterait également d'être abordé en termes d'efficacité, dans la mesure où il permet d'utiliser un savoir commun au service d'une narrativité minimale. Foncièrement monstratifs, les stéréotypes n'en agissent pas moins à la façon de " vecteurs narratifs ». En cela, ils constituent l'une des matières premières du régime des attractions, inscrivant par-là même les vues animées dans la tradition des institutions de divertissement populaire. 


\section{La femme et le type: le stéréotype comme vecteur narratif dans le cinéma des attractions}

\section{Pierre Chemartin et Nicolas Dulac}

\section{RÉSUMÉ}

Le cinéma des premiers temps avait largement recours aux stéréotypes, comme en témoigne l'abondance, au sein des vues animées, de figures féminines figées et simplistes. Plutôt que de l'appréhender seulement comme reflet d'une subjectivité, ainsi que le propose l'approche féministe traditionnelle, le stéréotype mériterait également d'être abordé en termes d'efficacité, dans la mesure où il permet d'utiliser un savoir commun au service d'une narrativité minimale. Foncièrement monstratifs, les stéréotypes n'en agissent pas moins à la façon de "vecteurs narratifs». En cela, ils constituent l'une des matières premières du régime des attractions, inscrivant par-là même les vues animées dans la tradition des institutions de divertissement populaire.

\section{ABSTRACT}

Early cinema routinely made use of stereotypes. In particular, the representation of women depended upon this tradition, with female figures regularly coded as recognizable types. Even though these stereotypes attest to a certain patriarchal vision within the new institution, they also employ common knowledge in the service of a minimal and cognitively induced narrativity. Highly monstrative, stereotypes also function as narrative vectors or "short cuts." For this very reason they took on an important role in the development of the "cinema of attractions," which occurred within the scope of many institutions of popular entertainment. 
Dans un catalogue Pathé de 1901, on peut lire, sous la rubrique "Scènes comiques", la description suivante: "Le vieux beau, qui se croit en train de dîner avec une demi-mondaine, se réveille dans les bras de son épouse.» Certains auront reconnu la vue de Ferdinand Zecca, Rêve et réalité, remake pratiquement identique du film Let Me Dream Again, réalisé par G. A. Smith l'année précédente. Ce synopsis, aussi concis soit-il, ne saurait décrire plus efficacement la vue. En fait, cette phrase ne décrit pas tant l'action du film qu'elle n'évoque à notre esprit une certaine "conjoncture» matrimoniale. Les personnages étant présentés à l'aide d'expressions génériques - le "vieux beau ", la «demi-mondaine", l' «épouse» —, ne sommes-nous pas amenés à en inférer, presque instinctivement, une situation de couple mille fois réactualisée? On imagine bien la scène: un couple vieillissant, le désir qui s'émousse, un mari aux prises avec le démon de midi, qui rêve d'aventures et de liberté. Par ailleurs, sans même avoir vu le film, on se représente facilement les personnages: un homme d'un certain âge, richement vêtu, hardi dans ses manières, et une jeune femme aux mœurs légères, aguichante à souhait. Puisqu'il s'agit d'une scène comique, il va de soi que l'épouse devra contraster avec la courtisane: on se la figurera bien évidemment laide, réfrigérante et vilainement habillée.

Destinés aux "exhibiteurs " et rédigés à des fins promotionnelles, les résumés contenus dans les catalogues sont censés traduire en peu de mots le contenu des vues. Cette description de Rêve et réalité ne déroge pas à la règle. Elle évoque, par ailleurs, un procédé récurrent dans le cinéma des débuts, en l'occurrence une utilisation presque instinctive de figures hautement stéréotypées en guise de personnages. Un échantillon limité de vues animées produites avant 1910 suffit à montrer que les "cinématographistes" de l'époque accordaient peu d'importance à l'élaboration psychologique et à l'approfondissement des traits de personnalité, et qu'ils préféraient mettre en scène des personnages "prédéfinis", aisément identifiables. On peut voir dans cette caractéristique du cinéma des premiers temps le corollaire de ce que Noël Burch (1990, p. 190) appelle l' "absence de persona classique», un trait fondamental du 
cinéma dit "primitif», qui découlerait principalement du jeu muet des acteurs et de l'éloignement de la caméra. Contraints à la simple extériorité, à "l'écriture de gestes", les personnages seraient ainsi vidés de leur "présence humaine" et devraient par conséquent mettre une croix sur toute velléité d'approfondissement psychologique. S’il est indéniable que le cinéma des premiers temps a une manière souvent fort simplificatrice de dépeindre ses personnages, il ne faudrait pourtant pas voir en cela le simple résultat de "contraintes" techniques, comme le laisse croire Burch. Au-delà des limitations du dispositif, il semble que le recours quasi systématique aux figures stéréotypales réponde avant tout à un souci d'efficacité, méthode qui fait par ailleurs écho à certaines stratégies déployées par les différentes institutions de divertissement au sein desquelles a émergé le cinéma ${ }^{1}$.

\section{L'approche cognitiviste du stéréotype et la théorie féministe}

À plusieurs égards, la représentation de la femme apparait comme un terrain d'investigation propice afin d'approfondir la notion de stéréotype dans le cinéma des premiers temps. Un simple coup d'œil aux catalogues de vente des diverses compagnies de l'époque suggère d'emblée la présence massive, dans les vues animées, de figures féminines stéréotypées. S'il s'avère fort pertinent pour l'étude des dimensions iconographiques et narratives du cinéma des premiers temps, le concept de stéréotype est toutefois encore l'objet de nombreux débats, qui rendent d'autant plus délicate son application théorique.

Cela n'a pourtant pas empêché l'étude du stéréotype de connaître, depuis les années 1930, un essor important dans de nombreuses disciplines, comme la psychanalyse, la sociologie et les études littéraires ${ }^{2}$. On doit entre autres cet essor théorique au journaliste Walter Lippman qui, en 1922, allait jeter sans le savoir les bases de l'approche cognitiviste du stéréotype avec un ouvrage intitulé Public Opinion. Dans le premier chapitre de son étude, "The World Outside and Pictures in Our Heads", Lippman (1997, p. 3-20) qualifiait les stéréotypes, précisément, d'«images dans nos têtes", expression promise à un bel avenir et qui allait devenir le point de départ d'une foule de réflexions sur 
le sujet (voir Ashmore et Del Boca 1981, p. 2). Contrairement aux tenants des approches socioculturelles ou psychodynamiques (voir Ashmore et Del Boca 1981), les cognitivistes n'envisagent plus le stéréotype de façon nécessairement péjorative, comme étant simplificateur ou figé (Amossy 1991, p. 50). Le recours aux stéréotypes relèverait plutôt d'une structure cognitive fondamentale, nous permettant de saisir l'information complexe venant de notre environnement et de la simplifier, afin de donner un sens au monde qui nous entoure. Ainsi, le stéréotype ne différerait pas des nombreuses constructions mentales façonnées par la collectivité et auxquelles a recours tout individu. En procédant par catégorisation, inférence, anticipation, une telle opération cognitive apparaît dès lors comme efficace et fonctionnelle. La façon dont ces schèmes cognitifs modèlent nos comportements peut bien évidemment donner lieu à la formation de préjugés socialement nocifs. Il n'en demeure pas moins, cependant, qu'avant d'être des outils de simplification idéologique ou morale, les stéréotypes permettent d'établir un savoir commun au sein d'une même population, comme en témoigne leur utilisation récurrente dans la plupart des productions culturelles propres à un lieu et à une époque.

La prise de conscience du stéréotype, que Ruth Amossy (1991, p. 21-48) situe dans la deuxième moitié du XIX siècle, coïncide d'ailleurs avec l'émergence d'une culture médiatique de masse, à laquelle participera sous peu le cinéma. Plusieurs manifestations de la culture populaire, telles que le vaudeville, la presse à sensation et le comic strip, misaient déjà, à cette époque, sur une utilisation récurrente de stéréotypes de tout acabit (liés à l'âge, à la profession, à la nationalité, au statut social, etc.), cherchant ainsi à maximiser leur portée et à faciliter leur interprétation. La nouveauté du cinéma, son insertion immédiate dans la sphère populaire ainsi que les limites imposées par son dispositif vont l'amener, au tournant du $\mathrm{Xx}^{\mathrm{e}}$ siècle, à faire usage, tout aussi spontanément, de figures stéréotypales. Tout individu qui se voit représenté dans les premières vues animées - que ce soit une femme, un homme, un étranger, etc. - sera presque toujours l'objet d'une catégorisation, d'une schématisation ostensible, qui peut parfois décontenancer le spectateur d'aujourd'hui. 
Cette simplification outrée des personnages, qui caractérise une bonne part du cinéma muet, a, conséquemment, ouvert la voie à des considérations d'ordre historique, socioculturel ou psychanalytique. Naturellement, les premières théories féministes se sont rapidement penchées sur cette dimension stéréotypale du cinéma, comme en témoignent les ouvrages précurseurs de Molly Haskell (1987) et de Marjorie Rosen (1973). Leurs travaux respectifs cherchaient à montrer comment, dès l'époque du muet, le cinéma a fétichisé et objectivé la femme en lui attribuant des rôles prédéfinis, en la dépeignant en fonction de types figés tels que la mère dévouée, la femme fatale, la pucelle ingénue, etc. En se penchant d'abord sur la représentation de la femme au sein du texte filmique, la théorie féministe qui a pris forme dans les années 1970 levait le voile sur un cinéma modelé en fonction de rôles sexuels hautement typés, et révélait du même coup ses structures idéologiques profondes. Cette approche, qui se situe au croisement de la théorie du dispositif, de la sémiologie et de la psychanalyse, montrait en quoi le cinéma, surtout celui issu des studios hollywoodiens, était façonné par un regard résolument masculin, un regard "fétichisant", où la femme, contrainte à la passivité, était dépeinte en tant qu'objet sexuel. Le cinéma des premiers temps s'est également présenté comme un objet propice à l'étude de ces procédés fondamentaux qui seront sous peu en usage dans le cinéma classique. Lucy Fisher, par exemple, passait au crible psychanalytique les films de Georges Méliès et les films à trucs en général, pour dégager l'«hostilité rampante à l'égard du sujet féminin " qui traverse les productions des premiers temps, mais aussi le cinéma en général (Fisher 1979).

Au courant des années 1980, cette vision a progressivement fait place à de nouvelles approches, davantage ancrées dans la réalité socioculturelle et l'espace de consommation du film. $S$ 'éloignant du principe premier voulant que les films proposent un regard résolument patriarcal et phallocentrique (vision dont font notamment état les travaux de Laura Mulvey ${ }^{3}$ ), ce nouveau regard féministe sur le cinéma ne se portait pas tant sur le texte que sur la relation du texte en question avec l'institution et le contexte culturel qui lui donnaient forme. Les recherches 
d'Annette Kuhn et, plus tard, de Miriam Hansen, Anne Friedberg, Giuliana Bruno ou Lauren Rabinovitz, par exemple, ne s'inscrivent plus dans un cadre politique ou idéologique prédéfini, mais cherchent à examiner la place qu'occupaient véritablement les femmes en tant que spectatrices dans l'espace d'exhibition, et à dégager la façon dont les films étaient interprétés par celles-ci ${ }^{4}$. La théorie du dispositif et la psychanalyse, qui ont inspiré les premières études féministes, faisaient état d'un sujet-spectateur théorique unitaire, nécessairement masculin, à la fois modelé par le texte et porteur des mêmes idéologies que ce dernier. Or, ce lien entre la sphère de production et la sphère de consommation est plus complexe qu'il n'y paraît. Patrice Petro (1994, p. 12-13) fait pertinemment remarquer que les écrits de Haskell et Rosen, pour lesquels le film reflète forcément une certaine image sociale de la femme, établissent de manière téléologique un rapport simplificateur entre texte et contexte. De nombreuses recherches portant sur le spectatorat féminin au temps du muet ont par ailleurs abondé dans ce sens, et ont démontré que l'on peut difficilement appréhender les modes de consommation filmique à l'aune d'une collectivité sociale rigide et uniforme, que la sphère spectatorielle est à la fois complexe, mouvante et hétérogène. Catherine Russell (2002), qui s'interroge dans un article récent sur la nature plurielle du spectatorat, remet en doute l'existence même, au sein des sites de consommation du film, de cette posture spectatorielle transcendante préconisée par le cinéma classique ${ }^{5}$.

La perspective développée ici, qui s'inspire, du moins dans ses prémisses, de l'approche cognitiviste, se présente comme un compromis entre une analyse textuelle et une analyse spectatorielle. En effet, il s'agit de voir en quoi le stéréotype, autant dans sa dimension visuelle que dans son potentiel narratif, sollicite un savoir collectif et propose, dans le cinéma des premiers temps, une clé d'interprétation préalable et cognitivement signifiante, une zone d'inférence sous-jacente à l'acte de consommation filmique nonobstant l'hétérogénéité de la sphère spectatorielle. Le stéréotype fait donc appel à un bagage socioculturel partagé par la très grande majorité des spectateurs 
et agit en tant que fondement interprétatif. En effet, comme dans notre propre rapport au monde, l'utilisation récurrente de stéréotypes dans les vues animées semble répondre à un souci d'efficacité qui, au cinéma, se manifestera aussi bien sur le plan monstratif que narratif. Puisqu'elle permet de comprendre la fonction du stéréotype en termes de rendement, autant chez l'individu que dans le groupe dont il fait partie, l'approche cognitive s'avère particulièrement appropriée, et ce, malgré ses évidentes limites. Bien qu'il ne s'agisse pas ici de s'engager dans une véritable étude "cognitiviste", les prémisses de cette approche peuvent servir de point de départ, dans la mesure où nous cherchons à comprendre en quoi les stéréotypes ont pu agir comme catalyseurs, comme "ferments actifs" dans le cinéma des premiers temps.

\section{Trois cas de figure : la vierge, la mère, la putain}

D’emblée, il semble que, dans les premières vues animées, la figure féminine coïncide avec une certaine "mystique féminine " (Friedan 1963), en l'occurrence cette tripartition classique de la femme: la vierge, la mère et la putain. Bien évidemment, ces stéréotypes agissent en quelque sorte comme des métacatégories, qui peuvent se présenter de diverses façons, donner lieu à de multiples variantes. La vierge apparaîtra sous les allures d'une belle jeune fille, douce, émotive et souvent offensée par les avances d'un homme un peu trop insistant, comme dans ces nombreuses vues animées où une servante doit résister aux propositions cavalières du maître de maison. Pensons à la vue The Washerwoman's Troubles, produite par Edison en 1897, dans laquelle une domestique est harcelée par un homme et finit par lui lancer un seau d'eau à la figure. De plus, la vierge est souvent représentée de manière à exalter l'innocence et la naïveté. C'est le cas de ces scènes où une ingénue s'affaire à quelque besogne quotidienne, faisant, par exemple, sa toilette matinale ou des exercices physiques, comme dans la vue The Pajama Girl, produite par la Biograph en 1903. D'une certaine façon, les nombreux tableaux vivants utilisent également cette figure de pureté et d'innocence ${ }^{6}$. Lorsqu'elle est plus âgée, la vierge prend des allures de «vieille fille». Dépourvue de ses attraits d'antan, elle 
est plutôt dépeinte comme une idiote et sera constamment la cible de facéties mesquines ou victime de quelque catastrophe provoquée par sa maladresse. Dans A Joke on the Old Maid (Biograph, 1901), une vieille domestique s'aperçoit que la lettre d'amour qu'elle vient de lire n'est en fait qu'un canular, ce qui la fait grimacer de manière grotesque.

La figure de la mère fournit également matière à de nombreux stéréotypes. La mère, mais aussi l'épouse, sera tantôt dépendante et soumise, tantôt laborieuse et dévouée, protectrice et maternelle, agressive et possessive. La mère est souvent représentée comme une personne qui accepte passivement son sort, malgré les conflits matrimoniaux, la violence conjugale, la pauvreté. Dans le film Buy Your Own Cherries (Paul's Animatograph, 1904), par exemple, l'épouse est maltraitée par son mari alcoolique et s'agrippe désespérément à lui pour l'empêcher de retourner à la taverne. La mère protectrice, quant à elle, veille à préserver la vertu de sa fille et au respect des bonnes manières, comme dans La fille mal et bien gardée (Gaumont, 1901) ou The Gay Shoe Clerk (Edison, 1903), ou défend son jeune enfant contre une menace quelconque, comme dans The Kidnapper (Biograph, 1903). Enfin, on ne dénombre pas les vues où l'épouse administre une violente raclée à son mari à la suite d'une infidélité ou d'un excès d'alcool, reproduisant ainsi le stéréotype de la femme opprimante et autoritaire. Un exemple parmi tant d'autres: Appointment by Telephone (Edison, 1902), où la femme surprend son mari au restaurant en compagnie de sa maîtresse et surgit dans la salle à manger pour le rosser brutalement. Bien qu'elle ne soit pas toujours aussi agressive, l'épouse s'avère généralement agaçante et querelleuse, au grand désagrément du mari. Ce dernier pourra éventuellement la faire taire en l'enfermant, par exemple, dans un lit pliant, comme c'est le cas dans Shut Up! (Biograph, 1902).

Si l'épouse est souvent trompée, elle commet aussi son lot d'infidélités. Généralement, dès que la sexualité de la femme entre en jeu, la putain prend le pas sur les deux autres figures. En effet, dans le cinéma des premiers temps, la sexualité s'exprime rarement au sein du mariage. Le désir féminin, puisqu'il ne se manifeste qu'en dehors de cette institution sacrée, 
est par conséquent associé au libertinage, à la dépravation et au vice, position que seule la putain est à même d'assumer. Aux côtés de la vierge et de la mère, donc, la figure de la putain contraste par son aspect éminemment sexué: elle véhicule des stéréotypes tels ceux de la femme aguicheuse, volage, séductrice. Elle aura souvent recours à des ruses fort inventives pour mieux berner son mari, comme dans Ménage à trois (Pathé, 1902) ou Courage de mari (Pathé, 1903). Dans ce dernier film, l'épouse cache habilement son amant sous la peau d'ours qui orne le sol de la chambre à coucher (Bousquet et Redi 1988, p. 91). Sa ruse, par ailleurs, lui donne souvent l'avantage sur l'homme enclin à ses penchants libidineux. Dans The Price of a Kiss (Biograph, 1902), par exemple, une coiffeuse se laisse embrasser par son client, après qu'un écriteau ait révélé qu'il en coûte un dollar de plus pour un tel service. Elle peut également devenir "mangeuse d'hommes» et est alors dépeinte comme étant narquoise, cruelle et insensible. Elle peut aussi conduire l'homme à sa perte, au risque d'être elle-même victime de ses vices; pensons, par exemple, à The Unfaithful Wife (Biograph, 1903), où une femme adultère ridiculise son mari avant d'être abattue par ce dernier, pris d'un excès de folie.

Comparée à la mère, la putain est représentée comme étant aimable et bienveillante. Sa sexualité assumée, par opposition à la sexualité réprimée de la mère-épouse, devient synonyme de plaisir, de frivolité et d'insouciance. L'exemple de Let Me Dream Again est, à cet égard, assez révélateur. Cette vue est orchestrée de manière à accentuer l'opposition qui distingue la figure de la mère et celle de la putain. Dans le rêve du protagoniste, d'abord, plusieurs aspects servent à affirmer le caractère avenant et désinvolte attribué à la figure de la putain: son costume on ne peut plus excentrique la situe d'emblée dans un espace irréel, presque fantasmatique, ce que souligne par ailleurs l'absence de décor en arrière-plan (figure 1). Son masque ajoute à son côté mystérieux et aguichant, masque que du reste elle enlève comme pour signifier une possible invitation d'ordre sexuel. L'alcool et la cigarette viennent ajouter à l'ambiance festive de débauche. Tout dans ce rêve renvoie au désir, à la complicité et aux épanchements lubriques. C'est au moment où l'homme se réveille 
aux côtés de sa femme que la figure de la putain prend son sens et que, réciproquement, la figure de la mère prend, elle aussi, un sens précis (figure 2). En effet, l'accoutrement de l'épouse et ses mimiques exagérées servent à accentuer son aspect repoussant et indésirable. L'opposition des deux figures suscite alors une réactualisation des stéréotypes qui rend plus patent le contraste entre rêve et réalité, renforçant ainsi l'efficacité du gag.

On constate que cette utilisation du stéréotype, aussi réductrice soit-elle, peut s'avérer efficace dans un mode de représentation naissant. La tripartition de la figure féminine, qui met en lumière ce procédé de stéréotypage adopté par le cinéma des débuts, participe d'une stratégie d'envergure qu'il ne faudrait pas réduire à quelque manifestation de la psyché masculine profonde. Cette stratégie s'inscrit dans une certaine mouvance qui apparaît dans différentes institutions de divertissement populaire, institutions qui ne valorisaient pas tant l'originalité du récit et son caractère édifiant — préconisés par les «belleslettres " ou le théâtre naturaliste, par exemple - que la dimension spectaculaire, la simple variation de situations bien connues, faisant partie du folklore ou des croyances populaires. D'ailleurs, les situations décrites ci-dessus ne sont en rien exclusives au cinéma, mais se retrouvent également dans le roman-feuilleton, le vaudeville, le comic strip, etc. L'usage du stéréotype est d'autant plus patent dans le cinéma naissant que celui-ci ne dispose pas encore d'un mode de production éprouvé ou de stratégies de mise en récit qui lui soient propres. Le stéréotype au cinéma, c'est en quelque sorte une stratégie par laquelle le spectaculaire "induit" le narratif, où le plaisir
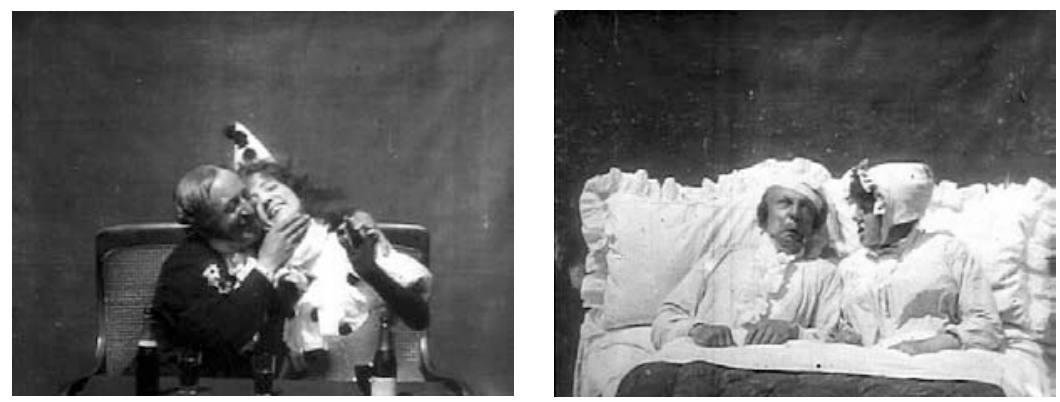

Figures 1 et 2. Let Me Dream Again (G.A. Smith, 1900) 
instantané et l'ébahissement priment sur l'identification et la contemplation.

\section{Le stéréotype dans la cinématographie-attraction}

Comme l'avançait Burch (1990), le recours aux stéréotypes tient, en partie, aux limitations imposées par le dispositif. Quoique ces "contraintes" n'aient pas été nécessairement perçues comme telles par les "cinématographistes» de l'époque - mais plutôt comme faisant partie d'un ensemble de procédures inhérentes au médium -, les stratégies de mise en image déployées dans les vues animées servent souvent à contourner, de diverses façons, les limites du dispositif (la courte durée des bandes, l'absence de son synchrone, la mobilité restreinte du point de vue, etc.). La concentration de l'action en une unité spatiotemporelle restreinte, le recours au bonimenteur, l'adaptation d'œuvres littéraires bien connues font, on le sait, partie intégrante de ce genre de stratégies, visant à assurer une bonne compréhension des vues par le spectateur.

L'emploi de stéréotypes, à cet égard, ne ferait-il pas partie de ce même ensemble de procédés? En facilitant à la fois la production et l'interprétation des vues, le stéréotype, au fond, peut être perçu comme un condensé de sens, un "prêt-à-porter de l'esprit" (Amossy 1991, p. 9), un objet au sein duquel s'amasse une charge sémantique qui dépasse largement la seule représentation. En effet, le "voir " qu'implique la fonction monstrative du cinéma est implicitement lié à un «savoir " prédéterminé et collectivement partagé. Les stéréotypes permettent ainsi d'utiliser un savoir commun au service d'une narrativité minimale, narrativité qui, du reste, n'est pas entièrement contenue dans la matière même du film, mais déduite par le spectateur. Condensée, ramassée sur elle-même, cette narrativité découle en effet d'une série d'idées embryonnaires et de conjectures, lesquelles sont suggérées par le truchement d'images hautement stéréotypées. Dès lors, ces stéréotypes sont réactualisés en fonction de "jalons" et de "schèmes" cognitifs culturellement marqués, renforçant par-là même la teneur narrative de la vue. Autrement dit, chaque stéréotype visuel, chaque cas de figure pour ainsi dire, ouvre un "champ de possibilités» dans 
lequel le spectateur puise pour doner un sens au récit. Ainsi en est-il de Let me Dream Again, où la narration, bien que minimale - elle tient davantage de la saynète ou du gag que du récit proprement dit —, s'ouvre à une narration de second niveau, qui surplombe en quelque sorte la dimension monstrative de la vue. Les stéréotypes, qui d'ailleurs s'appliquent également à la figure masculine, à la figure de l'étranger ou à celle de l'enfant, par exemple, agissent ici comme de véritables "vecteurs narratifs", sortes de raccourcis sémantiques facilitant l'acte de lecture en condensant le récit autour d'entités aisément identifiables. Linda Williams, en se penchant sur les procédés de différenciation sexuelle à l'œuvre dans les chronophotographies de Muybridge, y voyait déjà un procédé similaire. Dans les plaques chronophotographiques de Muybridge, la façon dont le corps de la femme est "mis en scène" et l'action dans laquelle elle est engagée font en sorte que celle-ci est fictionnalisée d'emblée, sa seule représentation provoquant «a diegetic surplus of meaning" (Williams, citée dans Balides 1998, p. 66).

À cet égard, il semble que le stéréotype ait un statut particulier dans le cinéma des premiers temps, dans la mesure où il témoigne d'une certaine perméabilité entre les deux «modes de pratiques filmiques» (Gaudreault et Gunning 1989, p. 57) a priori distincts que sont l'attraction et la narration. En premier lieu, le personnage qui véhicule les stéréotypes a une forte charge attractionnelle, puisqu'il a d'abord valeur en lui-même, par sa seule monstration. Effectivement, le stéréotype est porteur d'attributs ostensibles, directement perceptibles sur l'écran. L'attitude des personnages dans Let Me Dream Again, leurs mimiques exagérées, leur habillement et leurs gestes sont tout à fait représentatifs d'un tel régime de "confrontation exhibitionniste» (Gunning 1986, p. 66). Aussi réducteurs soient-ils, ces indices contribuent fortement à accentuer, à grossir les traits de ces figures (de ces types, devrions-nous dire) que sont celles $\mathrm{du}$ «vieux beau», de la «demi-mondaine» et de l'«épouse». Ils constituent, en quelque sorte, la matière première de la vue en tant que gag, et c'est par eux que s'expriment les stéréotypes. Les expressions du visage, tout comme les gestes, sont censés polariser l'attention sur des éléments significatifs de comportements 
prétendument masculins et féminins. Parce qu'allusifs, ces éléments iconographiques se réduisent fréquemment à quelques actions ou réactions fortement typées: le monsieur "cajole», la dame de ses rêves "minaude»; une fois éveillé, le même monsieur "grimace» et "se montre grincheux", l'épouse "indignée» le "gronde» et le "réprimande». Ces motifs n'ont de valeur que dans leur simplicité, peut-être même leur grossièreté. Leur fonction est de renvoyer, chez tout un chacun, à du déjà connu, et leur caractère fondamentalement attractionnel situe le propos dans une perspective atemporelle visant à donner une impression d'universalité.

En effet, les figures stéréotypales, puisqu'elles procèdent toujours d'une simplification, se présentent de manière à être immédiatement intelligibles, en misant beaucoup sur la schématisation, le caricatural ou le spectaculaire. La figure stéréotypale respecte donc la temporalité propre à l'attraction qui, selon Gunning (1996, p. 77), s'apparente à un "éclat de présence», à une "explosion soudaine": elle ne connaît que le "temps présent». De par leur charge attractionnelle, les figures stéréotypales véhiculées dans les vues animées ne supposent pas, d'emblée, une appréhension critique ou interprétative. La confrontation dont il est question dans le régime des attractions tient davantage du choc, de l'émerveillement et de la surprise. Cela pourrait laisser croire que le spectateur reste passif devant ce qu'il voit, que son rapport au film se limite à une stupéfaction improductive. C'est oublier que le cinéma des attractions, ou plutôt la "cinématographie-attraction ${ }^{7}$ ", fonctionne sur la base d'une coopération spectatorielle qui ne dépend pas d'une véritable mise en récit au sein de la vue, mais plutôt d'un savoir intertextuel apriorique. Comme l'a démontré Janet Staiger (1992, p. 101-123), les vues animées mettaient fréquemment en scène des événements de l'actualité récente ou des récits populaires bien connus, dont l'interprétation s'appuyait sur le savoir préalable du spectateur. Le stéréotype, en tant qu'agent de différenciation sociale, se présente lui aussi, et à plus forte raison encore, comme faisant partie du savoir collectif préalable au sein de la sphère spectatorielle. En effet, bien que la cinématographieattraction fonctionne avant tout sur une base essentiellement 
monstrative, certaines figures stéréotypales activent néanmoins un processus cognitif chez le spectateur, qui l'amène, premièrement, à reconnaître certains signes qui sous-tendent ce stéréotype et, ensuite, à leur inférer un sens à partir de connaissances préalablement acquises. C'est la capacité du stéréotype à opérer ce "glissement cognitif" de l'image vers un champ de possibles, du strictement visuel au potentiellement narratif, qui en fait un véritable vecteur de narration.

Puiser à même les stéréotypes qui circulent dans une société donnée permet donc d'activer un processus de narrativisation chez le spectateur, même si la vue ne cherche pas à créer cet effet. Certes, le stéréotype n'entre pas nécessairement dans le cadre d'une stratégie narrative, mais, en tant que figure spectaculaire et condensé sémantique, en tant que "voir" et "savoir", donc, le stéréotype joue à la fois sur les deux tableaux de l'attraction et de la narration. Dans Let Me Dream Again, deux «volets" suffisent à créer l'effet comique, effet qui repose non seulement sur un contraste visuel, mais aussi sur une opposition cognitivement induite entre les deux figures féminines. En fait, ce ne sont pas tant les différences physiques entre les deux femmes ou les grimaces de dégoût des deux époux éveillés qui provoquent le rire, que cette association cognitive qui conduit le spectateur à inférer toute une situation conjugale à partir de ce qu'il perçoit comme des stéréotypes: un vieux couple, un homme lassé de sa femme, une femme que son mari répugne, un ménage qui s'effrite et dans lequel la passion s'est visiblement épuisée. Autant de situations qui renvoient à des stéréotypes à partir desquels s'ouvre un monde de possibles, dans lequel le spectateur est libre d'aller puiser.

Le stéréotype, dans le cinéma des premiers temps, a donc une fonction particulière. Alors que, dans le cadre de nos rapports sociaux, le stéréotype a pour but de simplifier l'Autre, autrement dit de réaliser une réduction, dans les premières vues animées, il a plutôt pour fonction de produire une amplification. Dans la vie de tous les jours, quand l'individu a recours aux stéréotypes et à la catégorisation, il simplifie un environnement qu'il lui est impossible d'appréhender dans toute sa complexité. Par contre, dans les vues animées, le fabricant a recours aux stéréotypes afin 
de rendre avec efficacité un environnement qu'il lui est impossible de dépeindre dans toute sa complexité. Bien qu'il consiste lui aussi en une figure simplifiée, en une condensation de sens, le stéréotype que véhicule le cinéma des premiers temps sollicite un travail cognitif d'un ordre différent. Le spectateur aura pour tâche de "décondenser» le sens que les figures, à l'écran, s'attachent à réduire; il devra "amplifier» lui-même le sens contenu dans la matière filmique. Cet exercice fonctionne, pour l'essentiel, sur la base d'un travail de reconnaissance, travail qui permet d'associer une image à quelque vague reflet de la société dans laquelle le spectateur évolue. Ainsi, le spectateur peut reconnaittre dans un type un caractère à ce point familier qu'il l'identifiera à une figure bien connue de lui, peut-être même à quelqu'un de son entourage. Ces types, très largement exploités dans les différentes institutions de divertissement populaire, circulent toujours dans l'environnement social du spectateur. Parce qu'ils renvoient à son univers quotidien et contribuent grandement, par là même, à l'impressionner, les stéréotypes contenus dans les vues animées ont à la fois valeur de repères et de centres d'intérêt. Le régime des attractions monstratives suppose, nous l'avons dit, une certaine adhésion cognitive du spectateur, adhésion qui s'appuie sur un rapport de connivence entre fabricants et consommateurs de vues, au centre duquel se trouve la reconnaissance mutuelle de certaines figures conventionnelles et de certains schémas stéréotypaux.

\section{Réversibilité et subversion des stéréotypes}

L'utilisation du stéréotype dans la cinématographie-attraction soulève, en dernière analyse, des questions d'ordre idéologique. On peut se demander, notamment, si ces stéréotypes (aussi bien dans la société qu'à l'écran) sont révélateurs de la répression et de l'objectivation de la femme. Les théoriciennes féministes se sont déjà largement interrogées sur ces structures latentes d'oppression et sur leur signification historique et culturelle. Il semble toutefois que ce phénomène mérite aussi d'être analysé de façon plus pragmatique, notamment à l'aide de l'approche cognitiviste qui, sans nier les apports de la première vague de théories féministes, n'en jette pas moins un éclairage nouveau 
sur la question. À cet égard, le stéréotype s'est presque toujours présenté comme une notion de nature philosophique, idéologique ou psychanalytique, et rarement comme un agent sociocognitif. Dans son ouvrage The Woman at the Keyhole, Judith Mayne (1990, p. 159) étudie la manière dont le «mode d'adresse " du cinéma des premiers temps est modelé par une certaine dynamique des rôles sexuels et montre en quoi ce cinéma fait état de conceptions variées et opposées de l'altérité féminine. Or, cette multitude d' "autres" ne ferait-elle pas référence à autant de stéréotypes qui circulaient dans la société de l'époque? L'Autre peut être appréhendée non seulement à la lumière de quelque processus d'objectivation, mais également comme une catégorie sociale, déformée certes, mais non moins signifiante. Le stéréotype qui, par définition, ne s'applique qu’à l'Autre, pourrait ainsi être perçu comme un dispositif permettant à la collectivité de définir l'altérité, comme un outil qui, sans être dépourvu de toute signification idéologique, agit en premier lieu comme moyen d'interprétation et de différenciation sociale. Il faut, à tout le moins, être prudent lorsque vient le temps d'aborder le système de représentation du cinéma des premiers temps: avant de le considérer comme véhicule idéologique, ce qu'il est au demeurant, il faut, semble-t-il, l'aborder en tant que dispositif représentationnel efficace.

Faut-il rappeler que la "guerre" aux stéréotypes, aux clichés et aux idées reçues était déjà bien entamée au moment de l'émergence du cinéma. Alors que le roman historique et réaliste du début du XIX $X^{e}$ siècle laissait une grande place aux personnages stéréotypés, certains voyant même dans cette pratique quelque vertu littéraire, la seconde moitié du XIX siècle marque le début d'une "hantise " à l'égard du stéréotype, comme le démontre Ruth Amossy (1991, p. 65-73). À une époque d'explosion médiatique, qui voit prospérer le roman-feuilleton et la presse à sensation - époque qui correspond à l'industrialisation de la littérature —, l'élite bien pensante s'est dressée contre l'emprise de cette culture populaire et a refusé de s'y soumettre. Le cinéma, en adoptant des stéréotypes largement exploités dans la littérature grand public, mais aussi dans la presse, le vaudeville, le théâtre forain, s'engageait résolument dans les sillons de cette 
même culture populaire, au sein de laquelle l'usage des stéréotypes était pleinement assumé.

Avant qu'il ne s'institutionnalise, avant qu'il ne devienne un média autonome, le cinéma devait nécessairement compter sur une base interprétative commune à la grande majorité des spectateurs. Ainsi, les premiers "cinématographistes" n'inventaient ni ne faisaient la promotion de ces stéréotypes, mais les actualisaient, les exploitaient pour ainsi dire, par l'entremise du dispositif cinématographique. Déjà, ils pouvaient s'adonner à un jeu avec ces mêmes stéréotypes: les subvertir, les modifier, les confronter, en inverser les termes. D'une certaine façon, on peut avancer qu'à la manière du roman-feuilleton ou du vaudeville, le cinéma des premiers temps est subversif dans l'utilisation pleinement assumée qu'il fait des stéréotypes. Le stéréotype prend ici valeur d'icône, il est réduit à sa dimension monstrative. L'individualité, au fond, n'a pas sa place au sein du régime des attractions, elle ne peut qu'être inférée à partir d'un modèle. À maints égards, le stéréotype endosse la même fonction que les masques de la commedia dell'arte, en ce qu'il permet une identification rapide des personnages et de leurs caractéristiques fondamentales.

Dans Let Me Dream Again, tout n'est que mascarade et jeu - auxquels le masque de la demi-mondaine fait d'ailleurs directement référence —, tout n'est que comique élucubration autour d'un même prétexte narratif. Aux mimiques et aux gestes, déjà fort éloquents, s'ajoutent les décors, réduits à une collection d'objets épars (une table et des verres évoquent ici quelque vague débit de boissons; là, des oreillers, des draps, des bonnets de nuit évoquent une chambre à coucher) resserrant l'intrigue autour de lieux très facilement identifiables. La simplicité et l'efficacité de ces éléments visuels contribuent, au final, à refermer le propos sur lui-même, à masquer, précisément, tout effet de discours proprement idéologique. Il est permis, par là même, de ne voir dans cette farce qu'une simple mascarade, c'est-à-dire un étalage ostensible de clichés, dont l'aspect comique repose, précisément, sur le fait que le jeu des comédiens souligne exagérément l'aspect artificiel de leur performance. Les deux femmes, en effet, forcent sans ambages le 
trait des catégorisations sexuelles. L'une joue à la "demimondaine", l'autre joue à l' "épouse", de manière d'autant plus frappante que l'on cherche à insister sur la distinction entre "rêve" et "réalité». La vue, en tant que gag, prend son sens autour de ce contraste et dans le comportement fortement stéréotypé des deux figures féminines, de même, faut-il ajouter, que dans les réactions tout aussi stéréotypées et prévisibles de l'homme à l'égard des deux femmes.

Le cinéma des attractions, de ce fait, semble résister à toute forme de discours idéologique. Au fond, les vues animées seraient essentiellement tautologiques et rarement démonstratives, ce qui vient désamorcer la dimension péjorative associée au stéréotype. En analysant la représentation de l'étranger dans les vues réalisées par W. K. L. Dickson pour le kinétoscope (vues qui, à plusieurs égards, représentent le "degré zéro" de la cinématographie-attraction), Charles Musser (1997, p. 43) défendait une thèse similaire:

[The Kinetoscope films] offered a cosmopolitan vision that admittedly embraced stereotyping but generally avoided demeaning depictions [...]. Like the vaudeville stage $[\ldots]$, these films articulated a vision of American identity which was polyglot, self-confident and, within limits, fluid.

On peut tirer des conclusions semblables en ce qui concerne la représentation de la femme dans le cinéma des attractions en général. Bien que les vues animées dépeignent à larges traits des stéréotypes associés à la femme, ces derniers sont rarement narrativisés de manière à cristalliser une image dégradante et figée du rôle de la femme. La diversité et l'élasticité des stéréotypes offrent, une fois à l'écran, un panorama on ne peut plus hétéroclite de la société, un patchwork culturel et identitaire qui, à défaut d'être très "sophistiqué ", témoigne d'une volonté de n'épargner personne dans la caricature. En effet, tout comme dans le vaudeville, rares sont ceux qui ne se voient pas dépeints de manière outrancière dans les premières vues animées — qui ne se voient pas réduits à l'état de simples types. Du reste, l'image qui nous est donnée de l'homme dans les premières vues animées n'est guère plus "reluisante» que celle de la femme, 
celui-ci étant fréquemment représenté comme un rustre, un alcoolique, un coureur de jupons, etc. Dans Rêve et réalité, le "vieux beau» subit le même traitement simplificateur que les deux femmes et fait bien triste figure une fois paré de son bonnet, grimaçant de manière ridicule.

D'ailleurs, ce jeu sur les stéréotypes auquel s'adonne abondamment le cinéma des attractions est tout à fait manifeste dans le remake du film de Smith, Rêve et réalité, dont il a été question au tout début de cet article. Au premier coup d'œil, les deux vues paraissent assez semblables (figures 3 et 4). Dans le film de Zecca, cependant, l'épouse est visiblement interprétée par un homme, ce qui n'ajoute en rien à ses charmes. Mais il y a autre chose: la vieille dame ne s'offusque aucunement des élans de tendresse de son mari, élans du reste tout à fait accidentels puisque celui-ci rêve qu'il étreint une belle jeune femme. La réalité, par contraste, le surprend autant que nous, spectateurs. Repoussante, aux prises avec un mari visiblement lassé d'elle, l'épouse n'en a pas moins perdu ses ardeurs, contrairement à l'épouse de Let Me Dream Again. Le stéréotype de la femme victorienne "collet monté» voudrait en effet qu'une épouse de cet âge ne s'adonne plus à ce genre d'activité conjugale. Le fait que ce soit un homme qui interprète l'épouse opère un renversement qui fait appel à la complicité du spectateur et accentue l'effet du gag.

La vue Artistic Creation (Paul's Animatograph, 1901) opère aussi ce renversement du stéréotype. Cette vue est similaire à plusieurs films à trucs mettant en scène un prestidigitateur qui "fabrique» de toutes pièces une femme, comme c'est le cas dans
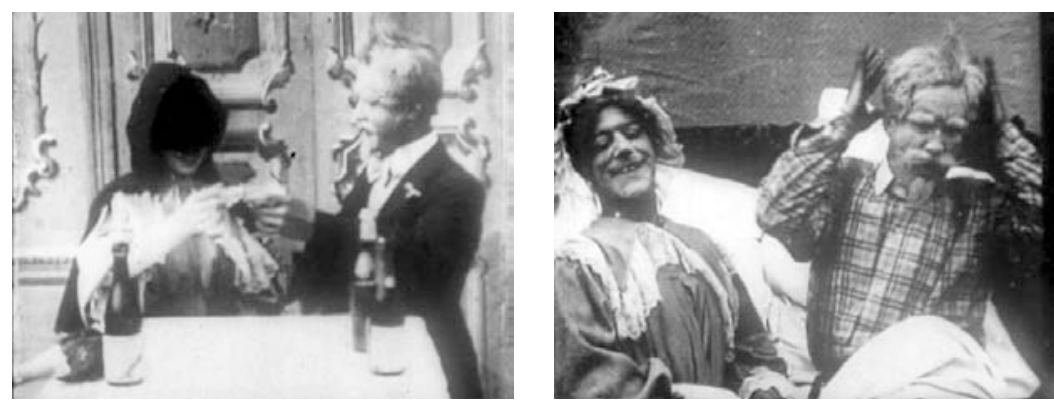

Figures 3 et 4. Rêve et réalité (Ferdinand Zecca, Pathé, 1901), droits réservés 
Reincarnation (Biograph, 1902). Dans la vue de Paul, le prestidigitateur dessine une à une les différentes parties du corps de la femme avant d'animer celle-ci. Une fois son ouvre terminée et la femme bien vivante, le magicien dessine alors — rien de plus naturel! — un bébé, qui prend vie à son tour. Or, contrairement à ce que voudrait le stéréotype de la mère aimante et dévouée, la femme repousse le bambin et s'enfuit, laissant le magicien seul avec l'enfant, qu'il tend, désabusé, en direction de la caméra. Paul, la même année, va encore une fois s'amuser à renverser ce stéréotype dans la vue An Over Incubated $B a b y$, dans laquelle une femme complètement exaspérée par sa progéniture demande les services d'un scientifique afin qu'il fasse grandir de manière accélérée le jeune enfant dans un incubateur géant.

On voit combien le stéréotype, par de légères variations, peut facilement être subverti afin de provoquer un effet comique de différents ordres. Ce faisant, les stéréotypes consacrés donnent lieu à une foule de variations, qui dénaturent considérablement les figures originales et désamorcent en partie la dimension péjorative qu'on leur accorde traditionnellement. À tel point, d'ailleurs, qu'on se demande si le terme "stéréotype", dont le préfixe stéréo signifie «solide», est toujours approprié.

En effet, les stéréotypes ne sont pas si figés qu'ils en ont l'air dans les premières années du cinéma. Cette particularité ne doit pas être négligée lorsque vient le temps d'étudier la représentation de la femme dans un média encore instable, qui puise à même les ressources d'institutions de divertissement qui nous sont relativement méconnues. De par la fonction particulière qui lui est attribuée, à la fois sur le plan monstratif et narratif, le stéréotype se voit accordée une place centrale dans le système de production et de consommation des vues animées. Avec l'institutionnalisation progressive du cinéma et l'émergence de codes de représentation spécifiques, les stéréotypes ayant trait à la femme se sont figés. À plusieurs égards, le cinéma des premiers temps paraît, comparativement, assez subversif, dans la mesure où celui-ci se permettait, en un temps où les conventions cinématographiques n'étaient pas encore établies, de remodeler constamment les stéréotypes, de les subvertir en quelque 
sorte. Si les vues animées ont parfois projeté des images de la femme moins heureuses que d'autres, voire déplorables, il n'en reste pas moins que c'est durant cette période que le recours aux stéréotypes semble le plus assumé, et par le fait même, le plus riche en variations de toutes sortes.

Comme Gustave Flaubert le suggérait en 1880 dans Bouvard et Pécuchet, la seule façon de se défaire des stéréotypes est peut-être de les étaler au grand jour, d'en faire une matière première, quitte à les pousser jusqu'à l'absurde, pour mieux en rire. C'est un peu ce que le premier cinéma s'évertuait spontanément à faire, un peu malgré lui peut-être, par souci d'efficacité sans doute.

\section{Université de Montréal}

\section{NOTES}

1. À cet égard, Molly Haskell (1987, p. 47) affirme: "To the extent that it prefers surfaces to essences and types to individuals, all of cinema is allegorical.» Or, il semble que le "typage» qui, pour Molly Haskell, caractérise le cinéma dans son ensemble, fonctionne différemment et réponde à d'autres exigences dans le cinéma des débuts qu'il ne le fait dans le cinéma classique plus tardif.

2. Les études consacrées aux stéréotypes, depuis les années 1950, sont nombreuses, surtout dans le domaine des sciences sociales. Ce phénomène ne se cantonne pas à la psychologie cognitive: certains penseurs, tels Theodor Adorno, Gordon Allport ou Erving Goffman se sont également penchés sur le problème. Voir, à cet effet, l'étude de Ruth Amossy et Anne Herschberg Pierrot (1997), qui fait le point sur les diverses approches du stéréotype en sciences sociales, dans les études littéraires et en linguistique. Citons, par ailleurs, l'article de Melinda Szaloky, paru récemment dans Cinémas, qui aborde les figures féminines et les stéréotypes à la lumière de la cognition sociale (Szaloky 2002).

3. Voir entre autres son important essai "Visual Pleasure and Narrative Cinema", paru en 1975 et reproduit dans Mulvey 1989 (p. 14-26).

4. Voir, entre autres, Kuhn 1982, Hansen 1991, Friedberg 1993, Bruno 1993 et Rabinovitz 1998.

5 «[... this unitary (male) subject [of classical film] may not even have existed outside the theoretical discourses of mass media and psychoanalytic semiotics " (Russell, 2002, p. 559).

6. Encore faut-il signaler que ces «tableaux vivants» sont porteurs d'une certaine ambiguïté. Chez Pathé, par exemple, les «naissances de Vénus» et autres "visions artistiques" sont profondément teintées d'érotisme et manifestement destinées à un public masculin. Ces vues sont par ailleurs classées sous la rubrique «vues grivoises d'un caractère piquant" (Bousquet et Redi 1988, p. 56).

7. Le terme "cinématographie-attraction" a été récemment proposé par André Gaudreault. Selon lui, cette expression témoigne avec plus de justesse du mode de pratique filmique des tout premiers temps du cinéma que l'expression consacrée "cinéma des attractions». Voir Gaudreault 2004. 


\section{RÉFÉRENCES BIBLIOGRAPHIQUES}

Amossy 1991 : Ruth Amossy, Les idées reçues. Sémiologie du stéréotype, Paris, Nathan, 1991.

Amossy et Herschberg Pierrot 1997 : Ruth Amossy et Anne Herschberg Pierrot, Stéréotypes et clichés. Langue, discours, société. Paris, Nathan, 1997.

Ashmore et Del Boca 1981 : Richard De Ashmore et Frances K. Del Boca, "Conceptual Approaches to Stereotypes and Stereotyping", dans David L. Hamilton (dir.), Cognitive Processes in Stereotyping and Intergroup Behavior, Hillsdale, Lawrence Erlbaum Associates, 1981, p. 1-35.

Balides 1998: Constance Balides, "Scenarios of Exposure in the Practice of Everyday Life: Women and the Cinema of Attractions", dans Annette Kuhn et Jackie Stacey (dir.), Screen Histories: A Screen Reader, Oxford, Clarendon Press, 1998.

Bousquet et Redi 1988 : Henri Bousquet et Riccardo Redi, «Pathé Frères. Les films de la production Pathé (1896-1914) 1", Quaderni di Cinema, n³ 37, 1988.

Bruno 1993: Giuliana Bruno, Streetwalking on a Ruined Map: Cultural Theory and the City Films of Elvira Notari, Princeton, Princeton University Press, 1993.

Burch 1990: Noël Burch, La lucarne de l’infini. Naissance du langage cinématographique, Paris, Nathan, 1990.

Fisher 1979: Lucy Fisher, "The Lady Vanishes: Women, Magic and the Movies", Film Quarterly, vol. 33, n 1 , 1979, p. 30-40.

Friedan 1963: Betty Friedan, The Feminine Mystique, New York, W.W. Norton \& Company, 1963.

Friedberg 1993: Anne Friedberg, Window Shopping: Cinema and the Postmodern, Berkeley, University of California Press, 1993.

Gaudreault 2004: André Gaudreault, Cinema delle origini, o della "cinematografiaattrazione», Milan, Castoro, 2004.

Gaudreault et Gunning 1989: André Gaudreault et Tom Gunning, «Le cinéma des premiers temps: un défi à l'histoire du cinéma?", dans Jacques Aumont, André Gaudreault et Michel Marie (dir.), L'histoire du cinéma. Nouvelles approches, Paris, Publications de la Sorbonne, 1989.

Gunning 1986 : Tom Gunning, "The Cinema of Attraction: Early Film, it's Spectator and the Avant Garde», Wide Angle, vol. 8, n 3-4, 1986.

Gunning 1996: Tom Gunning, "Now You See It, Now You Don't”: The Temporality of the Cinema of Attractions ", dans Richard Abel (dir.), Silent Film, London, Athlone, 1996.

Hansen 1991 : Miriam Hansen, Babel and Babylon: Spectatorship in American Silent Film, Cambridge, Harvard University Press, 1991.

Haskell 1987: Molly Haskell, From Reverence to Rape: The Treatment of Women in the Movies [1974], Chicago, University of Chicago Press, 1987.

Kuhn 1982 : Annette Kuhn, Women's Pictures: Feminism and Cinema, London, Routledge/Kegan Paul, 1982.

Lippman 1997 : Walter Lippman, Public Opinion [1922], New York, Free Press Paperbacks, 1997.

Mayne 1990 : Judith Mayne, The Woman at the Keyhole. Feminism and Women's Cinema, Bloomington, Indiana University Press, 1990.

Mulvey 1989: Laura Mulvey, Visual and Other Pleasures: Theories of Representation and Difference, Bloomington, Indiana University Press, 1989.

Musser 1997: Charles Musser, Edison Motion Pictures, 1890-1900: An Annotated Filmography, Gemona/Washington, Le Giornate del cinema muto/Smithsonian Institution Press, 1997. 
Petro 1994 : Patrice Petro, "Feminism and Film History», dans Diane Carson, Linda Dittmar et Janice R. Welsh (dir.), Multiple Voices in Feminist Film Criticism, Minneapolis, University of Minnesota Press, 1994.

Rabinovitz 1998: Lauren Rabinovitz, For the Love of Pleasure: Women, Movies, and Culture in Turn-of-the-Century Chicago, New Brunswick, Rutgers University Press, 1998.

Rosen 1973 : Marjorie Rosen, Popcorn Venus: Women, Movies and the American Dream, New York, Coward/McCann/Georghegan, 1973.

Russell 2002 : Catherine Russell, "Parallax Historiography. The Flâneuse as Cyberfeminist", dans Jennifer M. Bean et Diane Negra (dir.), A Feminist Reader in Early Cinema, Durham/London, Duke University Press, 2002, p. 552-570.

Staiger 1992: Janet Staiger, Interpreting Films: Studies in the Historical Reception of American Cinema, Princeton, Princeton University Press, 1992.

Szaloky 2002: Melinda Szaloky, "Silence Fiction: Rethinking (Under) Representations of the Feminine Through Social Cognition ", Cinémas, vol. 12, n 2, 2002.

Taylor 1981 : Shelley Taylor, "A Categorization Approach to Stereotyping", dans David L. Hamilton (dir.), Cognitive Processes in Stereotyping and Intergroup Behavior, Hillsdale, Lawrence Erlbaum Associates, 1981, p. 83-104. 